\title{
OPTIMAL CONTROL OF A NONLINEAR ELLIPTIC POPULATION SYSTEM
}

\author{
OVIDE ARINO ${ }^{1}$ AND JUAN-AURELIO MONTERO-SÁNCHEZ ${ }^{2}$ \\ ${ }^{1}$ IPRA, Département de Mathematiques 64000, Pau, France \\ ${ }^{2}$ Departamento de Análisis Matemático, \\ Universidad de Granada, 18071 Granada, Spain
}

(Received 15 December 1997; revised 26 September 1998)

\begin{abstract}
An optimal control for a nonlinear system is considered. The existence of an optimal-control pair, the characterization of the optimal control in terms of the optimal system and the uniqueness of solutions for the control problem are established. The uniqueness requires smallness assumptions on parameters in the functional.
\end{abstract}

Keywords: optimal control; nonlinear elliptic systems; existence; uniqueness; lower-upper solutions; strongly sublinear operators

AMS 1991 Mathematics subject classification: Primary 35B37; 35J65; 49K20; 49J20; 92D25

\section{Introduction}

We are going to study the problem

$$
\left.\begin{array}{cc}
-\Delta u=\sigma(x) v-d_{1}(x) u-c_{1} u(u+v), & \text { in } \Omega, \\
-\Delta v=b(x) u-d_{2}(x) v-c_{2} v(u+v), & \text { in } \Omega, \\
\frac{\partial u}{\partial \nu}=\frac{\partial v}{\partial \nu}=0, \quad \text { on } \partial \Omega, &
\end{array}\right\}
$$

where $\Omega$ is a bounded and regular domain in $\mathbb{R}^{N}$, the functions $\sigma, b, d_{1}, d_{2}$ belong to

$$
L_{+}^{\infty}(\Omega)=\left\{g \in L^{\infty}(\Omega), g \geqslant 0 \text { a.e. in } \Omega\right\},
$$

and $c_{1}, c_{2}$ are positive constants.

The coefficients of problem (1.1) can be interpreted as follows: we suppose that two subpopulations of the same species live in the domain $\Omega$. The function $u$ represents the concentration of the adult population and $v$ the young population. The function $\sigma$ describes the young that become adults. In the same way, $b$ is the rate of young produced by adults.

The functions $d_{1}$ and $d_{2}$ can be interpreted as harvesting a portion of the populations due to fishing. The constants $c_{1}$ and $c_{2}$ measure the strength of the crowding effect and 
the competition between $u$ and $v$. We are interested in finding solutions of system (1.1) with both components non-negative and non-trivial. For $i=1,2$ and $\delta_{i}>0$ we define

$$
C_{\delta_{i}}=\left\{g \in L^{\infty}(\Omega), \delta_{i} \geqslant g \geqslant 0 \text { a.e. in } \Omega\right\}
$$

The pair $\left(d_{1}, d_{2}\right) \in C_{\delta_{1}} \times C_{\delta_{2}}$ acts as a control on the fishery. For each value of the control in $C_{\delta_{1}} \times C_{\delta_{2}}$ (see Theorem 2.5 below), system (1.1) has a (weak) unique positive solution $\left(u_{d_{1}, d_{2}}, v_{d_{1}, d_{2}}\right)$.

Our cost-benefit functional is defined by $J: C_{\delta_{1}} \times C_{\delta_{2}} \rightarrow \mathbb{R}$, as

$$
J\left(d_{1}, d_{2}\right)=\int_{\Omega}\left\{\lambda u_{d_{1}, d_{2}}(x) d_{1}(x)-\left(d_{1}(x)\right)^{2}+\mu v_{d_{1}, d_{2}}(x) d_{2}(x)-\left(d_{2}(x)\right)^{2}\right\} \mathrm{d} x
$$

and in a certain sense represents the difference between revenues and cost (see $[\mathbf{2}, \mathbf{8}, \mathbf{1 0}, \mathbf{1 1}]$ for similar cost functionals). The positive constants $\lambda$ and $\mu$ stand for the ratio of the selling price of the fish to the cost of harvesting the populations (adults and young).

A control $\left(d_{1}, d_{2}\right) \in C_{\delta_{1}} \times C_{\delta_{2}}$ is said to be an optimal control if

$$
J\left(d_{1}, d_{2}\right)=\sup _{C_{\delta_{1}} \times C_{\delta_{2}}} J\left(e_{1}, e_{2}\right)
$$

The main result of this paper $(\S 3)$ is the uniqueness of the optimal control obtained basically when parameters $\lambda, \mu$ are small enough. Ideas explained here can be used to study the uniqueness of the optimal control in other problems $[\mathbf{8}, \mathbf{1 0}, \mathbf{1 1}]$.

In the scalar case, other types of conditions to obtain uniqueness of the optimal control can be found in [6].

In the next section, after some preliminary results, we give conditions to obtain uniqueness of the positive solution of (1.1).

In $\S 3$, we derive optimality conditions and, in particular, under certain hypotheses, each optimal control will be expressed in terms of the solution of an appropriate system (the optimality system).

In $\S 4$, by using the aforementioned optimality conditions, we get uniqueness of the optimal control. Also, a simple example is given in order to show that our conditions can be met.

\section{Existence of state solutions}

The upper- and lower-solutions method [1], and the uniqueness of positive solution for a strongly sublinear operator $[\mathbf{1}, \mathbf{9}]$, are the main tools used in this section (see Theorem 2.4 for a reminder of these notions). Also, it is convenient to use some results about the regularity of solutions for elliptic PDEs and embeddings of different Sobolev and Hölder spaces [7]. We will use $H^{1}(\Omega), H^{2}(\Omega), W^{1, p}(\Omega)$ and $W^{2, p}(\Omega)$ with $p \in(1, \infty)$ to denote the corresponding Sobolev spaces, and $C^{1, \alpha}(\Omega), \alpha \in(0,1)$ to represent the Hölder spaces. 
We will need to obtain conditions that give us existence and uniqueness for a linear system of type

$$
\left.\begin{array}{cc}
-\Delta \xi+a_{11}(x) \xi+a_{12}(x) \eta=f(x), & \text { in } \Omega, \\
-\Delta \eta+a_{21}(x) \xi+a_{22}(x) \eta=g(x), & \text { in } \Omega, \\
\frac{\partial \xi}{\partial \nu}=\frac{\partial \eta}{\partial \nu}=0, & \text { on } \partial \Omega,
\end{array}\right\}
$$

where $f, g \in L^{2}(\Omega)$.

We define $\rho_{1}(q)(\in \mathbb{R})$, for every $q \in L^{\infty}(\Omega)$, as the principal eigenvalue of the corresponding eigenvalue problem

$$
\left.\begin{array}{c}
-\Delta u+q u=\rho u, \quad \text { in } \Omega, \\
\frac{\partial u}{\partial \nu}=0, \quad \text { on } \Omega .
\end{array}\right\}
$$

It is known that $\rho_{1}(q)$ is simple and it verifies the variational characterization

$$
\rho_{1}(q)=\min _{u \in H^{1}(\Omega)-\{0\}} \frac{\int_{\Omega}|\nabla u|^{2}+\int_{\Omega} q u^{2}}{\int_{\Omega} u^{2}} .
$$

The function $\rho_{1}: L^{\infty}(\Omega) \rightarrow \mathbb{R}$ is continuous, $\rho_{1}\left(q_{1}\right) \geqslant \rho_{1}\left(q_{2}\right)$ if $q_{1} \geqslant q_{2}$ in $\Omega$, and $\rho_{1}(q+$ $M)=\rho_{1}(q)+M$ for $M \in \mathbb{R}$. One may define $\phi_{1}(q)$ to be the eigenfunction associated to $\rho_{1}(q)$ verifying $\phi_{1}(q)>0, \forall x \in \Omega$ and $\left\|\phi_{1}(q)\right\|_{\infty}=1$.

Proposition 2.1. Let $a_{11}, a_{12}, a_{21}, a_{22}$ be in $L^{\infty}(\Omega)$ and satisfying

$$
\rho_{1}\left(a_{11}\right)>0, \quad \rho_{1}\left(a_{22}\right)>0 \text { and }\left(\left\|a_{12}\right\|_{\infty}+\left\|a_{21}\right\|_{\infty}\right)^{2}<4 \rho_{1}\left(a_{11}\right) \rho_{1}\left(a_{22}\right) .
$$

Then, $\forall f, g \in L^{2}(\Omega)$, system (2.1) has a unique solution in $H^{1}(\Omega) \times H^{1}(\Omega)$.

Proof. It is enough to observe that the functional $A: H^{1}(\Omega) \times H^{1}(\Omega) \rightarrow \mathbb{R}$, defined as

$$
\begin{aligned}
A((\xi, \eta),(\varphi, \psi))=\int_{\Omega} \nabla \xi \nabla \varphi+\int_{\Omega} a_{11}(x) \xi \varphi & +\int_{\Omega} \nabla \eta \nabla \psi \\
& +\int_{\Omega} a_{22}(x) \eta \psi+\int_{\Omega} a_{12}(x) \eta \varphi+\int_{\Omega} a_{21}(x) \xi \psi
\end{aligned}
$$

is coercive, i.e. $\exists \alpha>0: A((\xi, \eta),(\xi, \eta)) \geqslant \alpha\left(|\xi|_{H^{1}(\Omega)}^{2}+|\eta|_{H^{1}(\Omega)}^{2}\right)$. In fact, by using the variational characterization of $\rho_{1}(q), q \in L^{\infty}(\Omega)$, and the Hölder inequality, we deduce that

$$
\begin{aligned}
A((\xi, \eta),(\xi, \eta)) & \\
& \geqslant \rho_{1}\left(a_{11}\right) \int_{\Omega} \xi^{2}+\rho_{1}\left(a_{22}\right) \int_{\Omega} \eta^{2}-\left(\left\|a_{12}\right\|_{\infty}+\left\|a_{21}\right\|_{\infty}\right) \int_{\Omega}|\xi \eta| \\
& \geqslant \rho_{1}\left(a_{11}\right)\|\xi\|_{L^{2}(\Omega)}^{2}+\rho_{1}\left(a_{22}\right)\|\eta\|_{L^{2}(\Omega)}^{2}-\left(\left\|a_{12}\right\|_{\infty}+\left\|a_{21}\right\|_{\infty}\right)\|\xi\|_{L^{2}(\Omega)}\|\eta\|_{L^{2}(\Omega)}
\end{aligned}
$$


The left-hand side, in the above inequality, is a quadratic form in the variables $\|\xi\|_{L^{2}(\Omega)}$ and $\|\eta\|_{L^{2}(\Omega)}$, and it will be positive defined if and only if (2.3) holds. Hence, there exists $\alpha_{1}>0$ such that

$$
A((\xi, \eta),(\xi, \eta)) \geqslant \alpha_{1}\left[\int_{\Omega} \xi^{2}+\eta^{2}\right] .
$$

On the other hand, from (2.3), one infers that $\exists \alpha_{2}>0$ satisfying

$$
\left[\left(1-\alpha_{2}\right) \rho_{1}\left(a_{11}\right)-\alpha_{2}\left\|a_{11}\right\|_{\infty}\right]\left[\left(1-\alpha_{2}\right) \rho_{1}\left(a_{22}\right)-\alpha_{2}\left\|a_{22}\right\|_{\infty}\right]>\frac{1}{4}\left(\left\|a_{12}\right\|_{\infty}+\left\|a_{21}\right\|_{\infty}\right)^{2},
$$

and, therefore,

$$
\begin{aligned}
{\left[\left(1-\alpha_{2}\right) \rho_{1}\left(a_{11}\right)-\alpha_{2}\left\|a_{11}\right\|_{\infty}\right]\|\xi\|_{L^{2}(\Omega)}^{2}+\left[\left(1-\alpha_{2}\right) \rho_{1}\left(a_{22}\right)-\alpha_{2}\left\|a_{22}\right\|_{\infty}\right]\|\eta\|_{L^{2}(\Omega)}^{2} } & \\
& -\left(\left\|a_{12}\right\|_{\infty}+\left\|a_{21}\right\|_{\infty}\right)\|\xi\|_{L^{2}(\Omega)}\|\eta\|_{L^{2}(\Omega)} \geqslant 0 .
\end{aligned}
$$

By virtue of the Hölder inequality, we have

$$
\begin{aligned}
\left(1-\alpha_{2}\right) \rho_{1}\left(a_{11}\right) \int_{\Omega} \xi^{2}+\left(1-\alpha_{2}\right) \rho_{1}\left(a_{22}\right) \int_{\Omega} \eta^{2}-\int_{\Omega}|\xi \eta|\left(\left\|a_{12}\right\|_{\infty}+\left\|a_{21}\right\|_{\infty}\right) & \\
& \geqslant \alpha_{2}\left(\left\|a_{11}\right\|_{\infty} \int_{\Omega} \xi^{2}+\left\|a_{22}\right\|_{\infty} \int_{\Omega} \eta^{2}\right) .
\end{aligned}
$$

Now, the variational characterization of $\rho_{1}\left(a_{11}\right), \rho_{1}\left(a_{22}\right)$ again implies

$$
\begin{aligned}
\left(1-\alpha_{2}\right)\left(\int_{\Omega}|\nabla \xi|^{2}+a_{11} \xi^{2}\right)+\left(1-\alpha_{2}\right)\left(\int_{\Omega}|\nabla \eta|^{2}+a_{22} \eta^{2}\right) & +\int_{\Omega} \xi \eta\left(a_{12}+a_{21}\right) \\
& \geqslant-\alpha_{2}\left(\int_{\Omega} a_{11} \xi^{2}+a_{22} \eta^{2}\right),
\end{aligned}
$$

or, equivalently,

$$
A((\xi, \eta),(\xi, \eta)) \geqslant \alpha_{2}\left[\int_{\Omega}|\nabla \xi|^{2}+|\nabla \eta|^{2}\right] .
$$

From (2.4), (2.6) and taking $\alpha=\frac{1}{2} \min \left\{\alpha_{1}, \alpha_{2}\right\}$, we deduce that $A$ is coercive.

The proof can be concluded by using the Lax-Milgram Lemma (for a similar proof see $[\mathbf{4}])$.

For each $e \in L^{\infty}(\Omega)$, we denote $\underline{e}=\operatorname{essinf}_{\Omega} e$ and, accordingly, $\bar{e}=\operatorname{esssup}_{\Omega} e$.

In the next theorem we give conditions to obtain a coexistence state.

Theorem 2.2. Let us assume that

$$
\left.\begin{array}{rl}
\underline{\sigma b} & >\delta_{1} \delta_{2}, \\
\bar{\sigma}-\underline{\sigma} & \leqslant \frac{c_{2} \underline{\underline{\sigma}}}{c_{1} \underline{\underline{b}}} \underline{\sigma}, \\
\bar{b}-\underline{b} & \leqslant \frac{c_{1} \underline{b}}{c_{2} \underline{\sigma}} \underline{b} .
\end{array}\right\}
$$


Then, $\forall\left(d_{1}, d_{2}\right) \in C_{\delta_{1}} \times C_{\delta_{2}}$, there exists a coexistence state $(u, v)$ of system (1.1), i.e. $(u, v)$ verifies system (1.1) with $u>0$ and $v>0$ in $\Omega$.

Proof. Firstly, let us consider system (1.1) with $\sigma, b, c_{1}$ and $c_{2}$ positive constants and $d_{1}, d_{2}$ non-negative constants. It is easy to check that system (1.1) has a unique constant solution $(u, v)$ such that $0<u<\left(\sigma / c_{1}\right), 0<v<\left(b / c_{2}\right)$ in $\Omega$, if and only if $\sigma b>d_{1} d_{2}$. In fact, $(u, v)$ is a solution, with positive constant components, of the system

$$
\begin{aligned}
& 0=\sigma v-d_{1} u-c_{1} u(u+v) \\
& 0=b u-d_{2} v-c_{2} v(u+v)
\end{aligned}
$$

Now, turning back to the general case, let $\left(u_{*}, v_{*}\right)$ be the unique positive constant solution of the problem

$$
\left.\begin{array}{rr}
-\Delta u=\underline{\sigma} v-\delta_{1} u-c_{1} u(u+v), & \text { in } \Omega \\
-\Delta v=\underline{b u}-\delta_{2} v-c_{2} v(u+v), & \text { in } \Omega \\
\frac{\partial u}{\partial \nu}=\frac{\partial v}{\partial \nu}=0, \quad \text { on } \partial \Omega . &
\end{array}\right\}
$$

Then $\left(u_{*}, v_{*}\right)$ is a subsolution of problem (1.1). In fact, $\left(u_{*}, v_{*}\right)$ satisfies the following inequalities:

$$
\begin{array}{cc}
-\Delta u_{*}=0 \leqslant \sigma v_{*}-d_{1} u_{*}-c_{1} u_{*}\left(u_{*}+v_{*}\right), & \text { in } \Omega, \\
-\Delta v_{*}=0 \leqslant b u_{*}-d_{2} u_{*}-c_{2} v_{*}\left(u_{*}+v_{*}\right), & \text { in } \Omega, \\
\frac{\partial u_{*}}{\partial \nu}=\frac{\partial v_{*}}{\partial \nu}=0 \leqslant 0, \quad \text { on } \partial \Omega . &
\end{array}
$$

Let us define $u^{*}=\left(\underline{\sigma} / c_{1}\right)$ and $v^{*}=\left(\underline{b} / c_{2}\right)$, which, by virtue of hypotheses of equation (2.7), satisfy

$$
\begin{gathered}
-\Delta u^{*}=0 \geqslant \sigma v^{*}-d_{1} u^{*}-c_{1} u^{*}\left(u^{*}+v^{*}\right), \quad \text { in } \Omega, \\
-\Delta v^{*}=0 \geqslant b u^{*}-d_{2} v^{*}-c_{2} v^{*}\left(u^{*}+v^{*}\right), \quad \text { in } \Omega, \\
\frac{\partial u^{*}}{\partial \nu}=\frac{\partial v^{*}}{\partial \nu}=0 \geqslant 0, \quad \text { on } \partial \Omega,
\end{gathered}
$$

and $u_{*} \leqslant u^{*}, v_{*} \leqslant v^{*}$ in $\Omega$. As an illustration, we will show the first inequality. In fact, the hypotheses of the theorem imply that

$$
\frac{\underline{b}}{c_{2}}(\sigma-\underline{\sigma}) \leqslant d_{1} \frac{\underline{\sigma}}{c_{1}}+\underline{\sigma}\left(\frac{\underline{\sigma}}{c_{1}}\right), \quad \forall x \in \Omega,
$$

and, consequently,

$$
0 \geqslant \sigma \frac{\underline{b}}{c_{2}}-d_{1} \frac{\underline{\sigma}}{c_{1}}-c_{1} \frac{\underline{\sigma}}{c_{1}}\left(\frac{\underline{\sigma}}{c_{1}}+\frac{\underline{b}}{c_{2}}\right), \quad \text { in } \Omega .
$$

Now, the upper-lower-solutions method and the regularity of the coefficients ensure the existence of a strictly positive solution $(u, v) \in\left(W^{2, p}(\Omega)\right)^{2}$ for $p \in(1, \infty)$. 
In fact, the system (1.1) can be rewritten in this way

$$
\left.\begin{array}{cc}
-\Delta u=\bar{f}(x, u(x), v(x)), & \text { in } \Omega, \\
-\Delta v=\bar{g}(x, u(x), v(x)), & \text { in } \Omega, \\
\frac{\partial u}{\partial \nu}=\frac{\partial v}{\partial \nu}=0, \quad \text { on } \partial \Omega,
\end{array}\right\}
$$

where $\bar{f}(x, u, v)=v\left(\sigma(x)-c_{1} u\right)-d_{1}(x) u-c_{1} u^{2}$ and $\bar{g}(x, u, v)=u\left(b(x)-c_{2} v\right)-d_{2}(x) v-$ $c_{2} v^{2}$, for all $x \in \Omega$.

Observe that function $\bar{f}$ is increasing in $v$ in the interval $v_{*} \leqslant v \leqslant v^{*}\left(=\underline{b} / c_{2}\right)$, for all $x \in \Omega, u_{*} \leqslant u \leqslant u^{*}\left(=\underline{\sigma} / c_{1}\right)$. Accordingly, function $\bar{g}$ is increasing in the interval $u_{*} \leqslant u \leqslant u^{*}$, for all $x \in \Omega, v_{*} \leqslant v \leqslant v^{*}$. So we have

$$
\begin{aligned}
& -\Delta u_{*} \leqslant \bar{f}\left(x, u_{*}, v_{*}\right), \quad \text { in } \Omega, \quad \frac{\partial u_{*}}{\partial \nu} \leqslant 0, \quad \text { on } \partial \Omega, \\
& -\Delta v_{*} \leqslant \bar{g}\left(x, u_{*}, v_{*}\right), \quad \text { in } \Omega, \quad \frac{\partial v_{*}}{\partial \nu} \leqslant 0, \quad \text { on } \partial \Omega,
\end{aligned}
$$

and

$$
\begin{aligned}
& -\Delta u^{*} \geqslant \bar{f}\left(x, u^{*}, v^{*}\right), \quad \text { in } \Omega, \quad \frac{\partial u^{*}}{\partial \nu} \geqslant 0, \quad \text { on } \partial \Omega \\
& -\Delta v^{*} \geqslant \bar{g}\left(x, u^{*}, v^{*}\right), \quad \text { in } \Omega, \quad \frac{\partial v^{*}}{\partial \nu} \geqslant 0, \quad \text { on } \partial \Omega \text {. }
\end{aligned}
$$

Choose $\Gamma>0$ such that the expression $\bar{f}(x, u, v)+\Gamma u$ is also increasing in $u \in\left[u_{*}, u^{*}\right]$, for all $x \in \Omega, v \in\left[v_{*}, v^{*}\right]$, and $\bar{g}(x, u, v)+\Gamma v$ is increasing in $v \in\left[v_{*}, v^{*}\right]$, for all $x \in \Omega$, $u \in\left[u_{*}, u^{*}\right]$. Now, by using 'maximum principle' arguments for the operator $-\Delta+\Gamma$ with Neumann boundary conditions and the monotonicity properties of $\bar{f}+\Gamma$ and $\bar{g}+\Gamma$ mentioned above, standard upper-lower-solutions type arguments complete the proof (see $[13]$ for details).

Now we need to prove the uniqueness of positive solution for system (1.1). For this purpose, the next operator is defined.

Definition 2.3. For every $v \in C(\bar{\Omega}) \cap L_{+}^{\infty}(\Omega)$, we will denote $P(v), P(v) \in C^{1, \alpha}(\bar{\Omega}) \subset$ $C(\bar{\Omega})$, the unique non-negative solution of the problem

$$
\left.\begin{array}{cc}
-\Delta u=\sigma(x) v-d_{1}(x) u-c_{1} u(u+v), & \text { in } \Omega, \\
\frac{\partial u}{\partial \nu}=0, \quad \text { on } \partial \Omega . &
\end{array}\right\}
$$

Theorem 2.4. The main properties of the operator $P$ are

(1) $P$ is well defined;

(2) if $v \in C(\bar{\Omega}) \cap L_{+}^{\infty}(\Omega), v \not \equiv 0 \Rightarrow P(v)>0$, in $\bar{\Omega}$; 
(3) $P$ is strongly sublinear, i.e. $\forall t \in(0,1), v \geqslant 0, v \neq 0 \Rightarrow P(t v)>t P(v)$; and

(4) under hypothesis (2.7), $P$ is monotone from the order-interval $\left[0,\left(\underline{b} / c_{2}\right)\right] \subset C(\bar{\Omega})$ into the order-interval $\left[0,\left(\underline{\sigma} / c_{1}\right)\right] \subset C(\bar{\Omega})$, i.e. $0 \leqslant u \leqslant v \Rightarrow P(u) \leqslant P(v)$, and

$$
0 \leqslant v \leqslant u \text { and } u \neq v \Rightarrow P(u)<P(v) \text { in } \bar{\Omega} .
$$

Proof. (1) Let us show that, for each $v \in C(\bar{\Omega}) \cap L_{+}^{\infty}(\Omega)$, problem (2.10) has one and only one solution $u$, which is such that

$$
u \in C^{1, \alpha}(\bar{\Omega}), \quad \forall \alpha \in(0,1) .
$$

In fact, if $v \equiv 0$, and $u \geqslant 0$, by the maximum principle, then $u \leqslant 0$, and, therefore, $u \equiv 0$. If $v \geqslant 0, v \neq 0$ in $\Omega$, observe that $u_{*} \equiv 0$ and $u^{*}=\bar{\sigma} / c_{1}$ are a lower solution and upper-solution (respectively) for (2.10). Now, let $w_{*}, w^{*} \in W^{2, p}(\Omega)$ be, for $p \in(1, \infty)$, the corresponding maximal and minimal solutions obtained by the upper-lower-solution method of (2.10) (see [1, Corollary 6.2]); consequently, $0 \leqslant w_{*} \leqslant w^{*} \leqslant u^{*}$ in $\Omega$. From the following inequality

$$
-\Delta\left(w^{*}-w_{*}\right)+c_{1} v\left(w^{*}-w_{*}\right) \leqslant 0
$$

and in view of the maximum principle, we obtain $w^{*} \leqslant w_{*}$. Observe that the solution $u=w_{*}=w^{*}$ satisfies $u \neq 0$ in $\Omega$, since $u \equiv 0$ is not a solution of problem (2.10).

We have shown the uniqueness of the solution in the strip $\left[0, u^{*}\right]$, but if we observe that any positive constant $M>u^{*}$ is an upper solution for problem $(2.10)$, then the same arguments as above give us the uniqueness in the general case.

(2) This is a consequence of the maximum principle, the regularity of $u\left(u \in C^{1, \alpha}(\bar{\Omega})\right.$, $\forall \alpha \in(0,1))$, and the Neumann boundary condition satisfied by $u$ (see $[7,12]$ ).

(3) Let us show that $P$ is a strongly sublinear map. Let $t \in(0,1), v \in C(\bar{\Omega}) \cap L_{+}^{\infty}(\Omega)$, $v \neq 0$. Then

$$
\begin{aligned}
-\Delta(t P(v)) & =t(-\Delta P(v))=t\left(\sigma v-d_{1} P(v)-c_{1}(P(v))^{2}-c_{1} v P(v)\right) \\
& =\sigma t v-d_{1} t P(v)-c_{1} t(P(v))^{2}-c_{1} t v P(v) \\
& <\sigma t v-d_{1} t P(v)-c_{1} t^{2}(P(v))^{2}-c_{1} t v P(v)
\end{aligned}
$$

and condition $\partial t P(v) / \partial \nu=0$ holds on $\partial \Omega$. From (2.11) we conclude that $t P(v)$ is a subsolution of problem (2.10) for $t v$. From the uniqueness of the non-negative solution of $(2.10), t P(v) \leqslant P(t v)$ in $\Omega$. Property (2) satisfied by $P$ yields the other part of (3).

(4) By virtue of (2.7) and $u$ satisfying the inequality $0 \leqslant u \leqslant\left(\underline{b} / c_{2}\right)$, we can take $\underline{\sigma} / c_{1}$ as a supersolution. Therefore, $0 \leqslant P(u) \leqslant\left(\underline{\sigma} / c_{1}\right)$. Now, observe that inequality $0 \leqslant u \leqslant v \leqslant\left(\underline{b} / c_{2}\right)$ implies that $P(u)$ is a subsolution for the problem of $P(v)$. In fact, we have

$$
\begin{aligned}
-\Delta P(u) & =u\left(\sigma-c_{1} P(u)\right)-d_{1} P(u)-c_{1} P(u)^{2} \\
& \leqslant v\left(\sigma-c_{1} P(u)\right)-d_{1} P(u)-c_{1} P(u)^{2} .
\end{aligned}
$$

The proof is completed by using the maximum principle. 
The main result of this section is the following theorem.

Theorem 2.5. Under hypothesis (2.7), problem (1.1) has a unique coexistence state in $\left[0,\left(\underline{\sigma} / c_{1}\right)\right] \times\left[0,\left(\underline{b} / c_{2}\right)\right]$.

Proof. One may consider the operator $Q$ analogous to $P$ such that $Q(u)=v$ is defined as the unique non-negative solution of

$$
\begin{gathered}
-\Delta v=b(x) u-d_{2}(x) v-c_{2} v(u+v), \quad \text { in } \Omega, \\
\frac{\partial v}{\partial \nu}=0, \quad \text { on } \partial \Omega .
\end{gathered}
$$

Now let us define $F: K \subset C(\bar{\Omega}) \rightarrow K$ as $F=Q \circ P$, here $K=\{w \in C(\bar{\Omega}): w \geqslant$ 0 in $\bar{\Omega}\}$. The operator $F$ has at least a non-trivial fixed point in $\left[0,\left(\underline{\sigma} / c_{1}\right)\right] \times\left[0,\left(\underline{b} / c_{2}\right)\right]$ via the coexistence state for the system (1.1), proved in Theorem 2.2. Conversely, any fixed point for the operator $F$ gives a coexistence state for system (1.1). By virtue of the above properties of operators $P$ and $Q$, the uniqueness of the positive fixed point of $F$ is given by the following theorem.

Theorem 2.6 (see [1, Theorem 24.2]). Let $(E, K)$ be an ordered Banach space whose positive cone has a non-empty interior. Suppose that $D$ is a star-shaped subset of $K$ containing 0 , and let $f: D \rightarrow E$ be a strongly sublinear and strongly increasing map. Then $f$ has at most one positive fixed point. Moreover, if $f(y)>y$ for some $y$, then $f$ has no positive fixed point in the order interval $[0, y]$.

\section{Existence of optimal control and optimality conditions}

In this section we show, under certain additional conditions, the existence of an optimal control and we describe some of the properties that such an optimal control verifies.

Theorem 3.1. Assume (2.7). Then the optimal-control problem has a solution, i.e. $\exists\left(d_{1}, d_{2}\right) \in C_{\delta_{1}} \times C_{\delta_{2}}$ such that $J\left(d_{1}, d_{2}\right)=\sup _{C_{\delta_{1}} \times C_{\delta_{2}}} J\left(e_{1}, e_{2}\right)$.

Proof. Observe that the functional $J$ is bounded from above. Let $s=\sup J$ and $\left(d_{1}^{n}, d_{2}^{n}\right)$ a maximizing sequence in $C_{\delta_{1}} \times C_{\delta_{2}}$. Then there exists a subsequence, again denoted by $\left(d_{1}^{n}, d_{2}^{n}\right)$, such that

$$
\left(d_{1}^{n}, d_{2}^{n}\right) \rightarrow\left(d_{1}^{*}, d_{2}^{*}\right) \in C_{\delta_{1}} \times C_{\delta_{2}} \text { weakly in } L^{2}(\Omega) \times L^{2}(\Omega)
$$

and

$$
\left(u^{n}, v^{n}\right) \equiv\left(u_{d_{1}^{n}, d_{2}^{n}}, v_{d_{1}^{n}, d_{2}^{n}}\right) \rightarrow\left(u^{*}, v^{*}\right) \text { strongly in } W^{1,2}(\Omega) \times W^{1,2}(\Omega) .
$$

In order to obtain the latter result, observe that the sequences $u^{n}, v^{n}$ are bounded in $W^{2,2}(\Omega)$, and, by virtue of the Rellich-Kondrachov Theorem, $W^{2,2}(\Omega)$ is compactly imbedded in $W^{1,2}(\Omega)$.

By a limit procedure in system (1.1), we obtain $u^{*}=u_{d_{1}^{*}, d_{2}^{*}}$ and $v^{*}=v_{d_{1}^{*}, d_{2}^{*}}$. 
Hence, by using the weakly lower semi-continuous property of the norm, we have

$$
\begin{aligned}
& s=\varlimsup \\
& =\int_{\Omega} \lambda u^{*} d_{1}^{*}+\mu v^{*} d_{2}^{*}+\varlimsup \lim \int_{\Omega}-\left(d_{1}^{n}\right)^{2}-\left(d_{2}^{n}\right)^{2} \leqslant J\left(d_{1}^{*}, d_{2}^{*}\right) .
\end{aligned}
$$

The next lemma gives a more precise upper bound for the optimal control.

Lemma 3.2. Suppose (2.7) and let $\lambda, \mu \in \mathbb{R}^{+}$in (1.2) such that

$$
\frac{\lambda \underline{\sigma}}{c_{1}}<\delta_{1}, \quad \frac{\mu \underline{b}}{c_{2}}<\delta_{2}
$$

If $\left(d_{1}, d_{2}\right)$ is an optimal control, then

$$
0 \leqslant d_{1} \leqslant \frac{\lambda \underline{\underline{\sigma}}}{c_{1}}, \quad 0 \leqslant d_{2} \leqslant \frac{\mu \underline{b}}{c_{2}}, \quad \text { in } \Omega
$$

Proof. Define $F=\min \left\{d_{1},\left(\lambda \underline{\sigma} / c_{1}\right)\right\}, G=\min \left\{d_{2},\left(\mu \underline{b} / c_{2}\right)\right\}$. We need to prove that

$$
J(F, G) \geqslant J\left(d_{1}, d_{2}\right)
$$

By taking $u_{F, G}$ and $v_{F, G}$ as upper solution and $u_{*}$ and $v_{*}$ as lower solution, the uniqueness of the positive solution of (1.1) implies $u_{F, G} \geqslant u_{d_{1}, d_{2}}, v_{F, G} \geqslant v_{d_{1}, d_{2}}$ in $\Omega$.

From the above inequalities we deduce that

$$
\begin{aligned}
J(F, G) & =\int_{\Omega} \lambda u_{F, G} F-F^{2}+\mu v_{F, G} G-G^{2} \\
& \geqslant \int_{\Omega} \lambda u_{d_{1}, d_{2}} F-F^{2}+\mu v_{d_{1}, d_{2}} G-G^{2}
\end{aligned}
$$

We need to prove that

$$
\int_{\Omega} \lambda u_{d_{1}, d_{2}} F-F^{2} \geqslant \int_{\Omega} \lambda u_{d_{1}, d_{2}} d_{1}-d_{1}^{2}
$$

In fact, in $\Omega_{1}=\left\{x \in \Omega: d_{1}>\left(\lambda \underline{\sigma} / c_{1}\right)\right\}$ we get $\lambda u_{d_{1}, d_{2}} \leqslant\left(\lambda \underline{\sigma} / c_{1}\right) \leqslant d_{1}+F$, and, therefore,

$$
-\lambda u_{d_{1}, d_{2}}\left(-F+d_{1}\right)+\left(-F+d_{1}\right)\left(d_{1}+F\right) \geqslant 0 \Rightarrow \lambda u_{d_{1}, d_{2}} F-F^{2} \geqslant \lambda u_{d_{1}, d_{2}} d_{1}-d_{1}^{2} .
$$

On the other hand, in $\Omega-\Omega_{1}$ we have $\lambda u_{d_{1}, d_{2}} F-F^{2}=\lambda u_{d_{1}, d_{2}} d_{1}-d_{1}^{2}$.

Analogously,

$$
\mu v_{d_{1}, d_{2}} G-G^{2} \geqslant \mu v_{d_{1}, d_{2}} d_{2}-d_{2}^{2}, \quad \text { in } \Omega,
$$

and the proof is complete. 
To obtain the optimality conditions, we need to differentiate the functional with respect to the controls. Since the state solutions are explicitly in the functional and do depend on the controls, we must differentiate the state solutions with respect to the controls.

Proposition 3.3. Suppose (2.7). Let $\left(u_{*}, v_{*}\right)$ be the solution of system (2.8) and assume that the following inequality holds

$$
\left(\bar{\sigma}-c_{1} u_{*}+\bar{b}-c_{2} v_{*}\right)^{2}<4 \rho_{1}\left(d_{1}+c_{1}\left(2 u_{*}+v_{*}\right)\right) \rho_{1}\left(d_{2}+c_{2}\left(u_{*}+2 v_{*}\right)\right),
$$

where $\left(d_{1}, d_{2}\right) \in C_{\delta_{1}} \times C_{\delta_{2}}$ is an admissible control and $f, g \in L^{\infty}(\Omega)$ are such that $\left(d_{1}+\beta f, d_{2}+\beta g\right) \in C_{\delta_{1}} \times C_{\delta_{2}}$, for $\beta>0$ small enough. Let us denote $\left(u_{d_{1}, d_{2}}, v_{d_{1}, d_{2}}\right)$ as $(u, v)$ and $\left(u_{d_{1}+\beta f, d_{2}+\beta g}, v_{d_{1}+\beta f, d_{2}+\beta_{g}}\right)$ as $\left(u_{\beta}, v_{\beta}\right)$. Then the functions

$$
\begin{array}{ll}
\frac{u_{\beta}-u}{\beta} \rightarrow \xi, & \text { in } H^{1}(\Omega), \\
\frac{v_{\beta}-v}{\beta} \rightarrow \eta, & \text { in } H^{1}(\Omega),
\end{array}
$$

as $\beta \searrow 0$, where $(\xi, \eta)$ is the unique solution of the following system:

$$
\left.\begin{array}{cc}
-\Delta \xi+\left[d_{1}+c_{1}(2 u+v)\right] \xi-\left(\sigma-c_{1} u\right) \eta=-f u & \text { in } \Omega \\
-\Delta \eta+\left[d_{2}+c_{2}(u+2 v)\right] \eta-\left(b-c_{2} v\right) \xi=-g v, & \text { in } \Omega \\
\frac{\partial \xi}{\partial \nu}=\frac{\partial \eta}{\partial \nu}=0, \quad \text { on } \partial \Omega . &
\end{array}\right\}
$$

Proof. One may check that the pair $\left(\xi_{\beta}, \eta_{\beta}\right)$ defined by

$$
\xi_{\beta}=\frac{u_{\beta}-u}{\beta}, \quad \eta_{\beta}=\frac{v_{\beta}-v}{\beta}
$$

is the unique solution of the following system:

$$
\left.\begin{array}{cc}
-\Delta \xi_{\beta}+\left[d_{1}+c_{1}\left(u+u_{\beta}+v\right)\right] \xi_{\beta}-\left(\sigma-c_{1} u_{\beta}\right) \eta_{\beta}=-f u_{\beta}, & \text { in } \Omega \\
-\Delta \eta_{\beta}+\left[d_{2}+c_{2}\left(u+v+v_{\beta}\right)\right] \eta_{\beta}-\left(b-c_{2} v_{\beta}\right) \xi_{\beta}=-g v_{\beta}, & \text { in } \Omega, \\
\frac{\partial \xi_{\beta}}{\partial \nu}=\frac{\partial \eta_{\beta}}{\partial \nu}=0, \quad \text { on } \partial \Omega . &
\end{array}\right\}
$$

By Proposition 2.1, $\exists M>0$ such that $\left|\xi_{\beta}\right|_{H^{1}},\left|\eta_{\beta}\right|_{H^{1}} \leqslant M$ independently on $\beta$. Using elliptic estimates one can see that boundedness actually extends to $H^{2}(\Omega)$. For any sequence $\beta_{n} \searrow 0$, there exists a subsequence (again denoted by $\beta_{n}$ ) such that $\xi_{n} \rightarrow \xi$ and $\eta_{n} \rightarrow \eta$ in $H^{1}(\Omega)$, with $(\xi, \eta)$ the unique solution of the above system (3.5). The uniqueness of $(\xi, \eta)$ assures the convergence of the whole sequence.

Remark 3.4. Using the properties of $\rho_{1}$, the above proposition holds true by replacing (3.4) with

$$
\left(\bar{\sigma}-c_{1} u_{*}+\bar{b}-c_{2} v_{*}\right)^{2}<4 c_{1} c_{2}\left(2 u_{*}+v_{*}\right)\left(u_{*}+2 v_{*}\right)
$$


We will now derive optimality conditions. Also, we will use here the notation $w^{+}(x)=$ $\sup \{w(x), 0\}$ for $x \in \Omega$.

Theorem 3.5. Assume (2.7) and (3.1). If $\left(d_{1}, d_{2}\right) \in C_{\delta_{1}} \times C_{\delta_{2}}$ is an optimal control satisfying (3.4), then

$$
\left.\begin{array}{ll}
d_{1}=\frac{1}{2} \lambda u(1-r)^{+}, & \text {in } \Omega, \\
d_{2}=\frac{1}{2} \mu v(1-s)^{+}, & \text {in } \Omega,
\end{array}\right\}
$$

where $(u, v)$ is the solution of (1.1) and $(r, s)$ is the solution of the adjoint system

$$
\left.\begin{array}{c}
-\Delta r+\left[d_{1}+c_{1}(2 u+v)\right] r-\frac{\mu}{\lambda}\left(b-c_{2} v\right) s=d_{1}, \quad \text { in } \Omega, \\
-\Delta s+\left[d_{2}+c_{2}(u+2 v)\right] s-\frac{\lambda}{\mu}\left(\sigma-c_{1} u\right) r=d_{2}, \quad \text { in } \Omega, \\
\frac{\partial r}{\partial \nu}=\frac{\partial s}{\partial \nu}=0, \quad \text { on } \partial \Omega .
\end{array}\right\}
$$

Proof. Note that, under the hypotheses of the theorem, system (3.9) has a unique solution (observe that the system verified by $(R, S)=(\lambda r, \mu s)$ is of type (3.5)). Let $f, g \in L^{\infty}(\Omega)$ be such that $\left(d_{1}+\beta f, d_{2}+\beta g\right) \in C_{\delta_{1}} \times C_{\delta_{2}}$ as $\beta \searrow 0$. By using that $\left(d_{1}, d_{2}\right)$ is an optimal control, we have

$$
J\left(d_{1}, d_{2}\right) \geqslant J\left(d_{1}+\beta f, d_{2}+\beta g\right)
$$

if and only if

$$
\begin{aligned}
\int_{\Omega} \lambda\left(u_{\beta}-u\right)\left(d_{1}+\beta f\right)+\mu\left(v_{\beta}-v\right)\left(d_{2}\right. & +\beta g)+\lambda \beta u f \\
& +\mu \beta v g+\int_{\Omega}-2 \beta d_{1} f-2 \beta d_{2} g-\beta^{2} f^{2}-\beta^{2} g^{2} \leqslant 0 .
\end{aligned}
$$

Dividing by $\beta>0$ and taking $\beta \searrow 0$,

$$
\int_{\Omega} \lambda \xi d_{1}+\mu \eta d_{2}+\lambda u f+\mu v g-2 d_{1} f-2 d_{2} g \leqslant 0 .
$$

Multiplying in (3.9) the first equation by $\lambda \xi$, the second by $\mu \eta$, and integrating on $\Omega$

$$
\begin{aligned}
\int_{\Omega} \lambda \nabla r \nabla \xi+ & {\left[d_{1}+c_{1}(2 u+v)\right] \lambda r \xi-\left(b-c_{2} v\right) \xi \mu s } \\
& +\int_{\Omega} \mu \nabla s \nabla \eta+\left[d_{2}+c_{2}(2 v+u)\right] \mu s \eta-\left(\sigma-c_{1} u\right) \lambda r \eta=\int_{\Omega} \lambda d_{1} \xi+\int_{\Omega} \mu d_{2} \eta
\end{aligned}
$$

By similar arguments with $\lambda r$ and $\mu s$ in (3.5), we obtain

$$
\begin{aligned}
\int_{\Omega} \lambda \nabla r \nabla \xi & +\left[d_{1}+c_{1}(2 u+v)\right] \lambda r \xi-\left(b-c_{2} v\right) \xi \mu s \\
& +\int_{\Omega} \mu \nabla s \nabla \eta+\left[d_{2}+c_{2}(2 v+u)\right] \mu s \eta-\left(\sigma-c_{1} u\right) \lambda r \eta=-\int_{\Omega} f u \lambda r-\int_{\Omega} g v \mu s
\end{aligned}
$$


hence

Now, from (3.10) we have

$$
\int_{\Omega} \lambda d_{1} \xi+\int_{\Omega} \mu d_{2} \eta+\int_{\Omega} f u \lambda r+\int_{\Omega} g v \mu s=0
$$

$$
\int_{\Omega}-f u \lambda r-g v \mu s+\lambda u f+\mu v g-2 d_{1} f-2 d_{2} g \leqslant 0 .
$$

Taking $g \equiv 0,(3.11)$ implies

$$
\int_{\Omega} f\left[-\lambda u r+\lambda u-2 d_{1}\right] \leqslant 0
$$

If $f \in L_{+}^{\infty}(\Omega)$, from (3.1) (which implies $\left|d_{1}\right|_{\infty}<\delta_{1}$ ) one deduces that there exists $\beta_{0}>0$ such that $d_{1}+\beta f \in C_{\delta_{1}}$, for $0 \leqslant \beta<\beta_{0}$. For this reason, $2 d_{1} \geqslant \lambda u(1-r)$ and, equivalently,

$$
d_{1} \geqslant \frac{1}{2} \lambda u(1-r), \quad \text { in } \Omega .
$$

Now, by taking $f=-d_{1}$ and $\beta<1$, one infers that

$$
\int_{\Omega} d_{1}\left(\lambda u(1-r)-2 d_{1}\right) \geqslant 0 .
$$

Hence, we have

$$
\begin{aligned}
& d_{1}=\frac{1}{2} \lambda u(1-r), \quad \text { in } \Omega \cap\left\{d_{1}>0\right\} \\
& d_{1} \geqslant \frac{1}{2} \lambda u(1-r), \quad \text { in } \Omega .
\end{aligned}
$$

In conclusion,

$$
d_{1}=\frac{1}{2} \lambda u(1-r)^{+}, \quad \text { in } \Omega,
$$

and, analogously,

$$
d_{2}=\frac{1}{2} \mu v(1-s)^{+}, \quad \text { in } \Omega \text {. }
$$

Corollary 3.6. Under the hypotheses of the previous theorem, if $\left(d_{1}, d_{2}\right) \in C_{\delta_{1}} \times C_{\delta_{2}}$ is an optimal control, then

$$
\begin{aligned}
& d_{1}=\frac{1}{2} \lambda u(1-r)^{+}, \quad \text { in } \Omega, \\
& d_{2}=\frac{1}{2} \mu v(1-s)^{+}, \quad \text { in } \Omega,
\end{aligned}
$$

where $(u, v, r, s)$ is a solution of the optimality system

$$
\left.\begin{array}{c}
-\Delta u=v\left(\sigma-c_{1} u\right)-u^{2}\left[\frac{1}{2} \lambda(1-r)^{+}+c_{1}\right], \quad \text { in } \Omega, \\
-\Delta v=u\left(b-c_{2} v\right)-v^{2}\left[\frac{1}{2} \mu(1-s)^{+}+c_{2}\right], \quad \text { in } \Omega, \\
-\Delta r+c_{1}(2 u+v) r-\frac{\mu}{\lambda}\left(b-c_{2} v\right) s=\frac{1}{2} \lambda u\left[(1-r)^{+}\right]^{2}, \quad \text { in } \Omega, \\
-\Delta s+c_{2}(u+2 v) s-\frac{\lambda}{\mu}\left(\sigma-c_{1} u\right) r=\frac{1}{2} \mu v\left[(1-s)^{+}\right]^{2}, \quad \text { in } \Omega, \\
\frac{\partial u}{\partial v}=\frac{\partial v}{\partial v}=\frac{\partial r}{\partial v}=\frac{\partial s}{\partial v}=0, \quad \text { on } \partial \Omega,
\end{array}\right\}
$$


and

$$
0<u<\frac{\underline{\sigma}}{c_{1}}, \quad 0<v<\frac{\underline{b}}{c_{2}}
$$

\section{Uniqueness of the optimal control}

In order to prove the uniqueness of the optimal control, we need to deduce some additional properties of optimal controls.

Observe that, as a consequence of Proposition 3.3 and Theorem 3.5, in the previous section the Gateaux differentiability of $J$ was proven.

We will prove that if $\lambda, \mu$ are small enough then $\left(d_{1}, d_{2}\right) \in \int\left(C_{\delta_{1}} \times C_{\delta_{2}}\right)$ and functional $J$ is Frechet continuously differentiable provided that $\left(d_{1}, d_{2}\right)$ is an optimal control. The uniqueness of the optimal control follows from a convexity argument (see [3,5] as general references).

Lemma 4.1. Suppose (2.7), $\left(u_{*}, v_{*}\right)$ a positive solution of system (2.8) and $k_{1} \lambda<$ $\mu<k_{2} \lambda$ for $k_{1}, k_{2}$ fixed positive constants. Let $\left(d_{1}, d_{2}\right)$ be an optimal control satisfying (3.4). Then there exist $\lambda_{0}, \mu_{0}>0$, such that the inequalities

$$
\begin{aligned}
& \frac{1}{4} \lambda u_{*} \leqslant d_{1} \leqslant \lambda \frac{\underline{\sigma}}{c_{1}}, \quad \text { in } \Omega, \\
& \frac{1}{4} \mu v_{*} \leqslant d_{2} \leqslant \mu \frac{\underline{b}}{c_{2}}, \quad \text { in } \Omega,
\end{aligned}
$$

hold true for $0<\lambda<\lambda_{0}$ and $0<\mu<\mu_{0}$.

Proof. Take $\lambda, \mu$ satisfying (3.1). Then, from Theorem 3.5, $\left(d_{1}, d_{2}\right)$ verifies (3.8). Hence the proof is completed by showing that $r, s<\frac{1}{2}$ in $\Omega$ when $\lambda, \mu$ are small enough. From the proof of Proposition 2.1, one deduces that there exists a positive constant $c$ such that $(R, S)=(\lambda r, \mu s)$ satisfying

$$
|R|_{H^{1}(\Omega)}<c \lambda^{2}, \quad|S|_{H^{1}(\Omega)}<c \mu^{2} .
$$

Again, by elliptic regularity $|r|_{\infty}<c \lambda,|s|_{\infty}<c \mu$ (here $c$ denotes different constants in each case). This last fact is a consequence of the Rellich-Kondrachof Theorem and the elliptic equations verified by $r$ and $s$. If $N \leqslant 2$, by the arguments mentioned above, $r, s \in$ $L^{p}(\Omega)$ for $p \in(1, \infty)$, in particular for $p \in(N, \infty)$. So, $r, s \in W^{1, p}(\Omega) \hookrightarrow C^{0, \alpha}(\bar{\Omega})$ for $\alpha=1-(N / p)$. On the other hand, if $N>2, r, s \in H^{1}(\Omega) \hookrightarrow L^{r_{1}}(\Omega)$, with $r_{1}=2 N /(N-$ 2) $>2=r_{0}$. In this way, as $-\Delta r+\left[d_{1}+c_{1}(2 u+v)\right] r=(\mu / \lambda)\left(b-c_{2} v\right) s+d_{1} \in L^{r_{1}}(\Omega)$, then $r \in W^{1, r_{1}}$ and, analogously, $s \in W^{1, r_{1}}$. If $r_{1} \leqslant N$, again the Rellich-Kondrachov Theorem finishes the proof. If $r_{1}<N$, then we can repeat the same process and we will obtain $n_{0}$ such that $r_{n_{0}} \geqslant N$, since $\left\{r_{n}\right\}<N, \forall n \in \mathbb{N}$, yields a contradiction.

Proposition 4.2. Under hypotheses (2.7) and (3.7) and taking $0<\lambda<\lambda_{0}, 0<\mu<$ $\mu_{0}\left(\lambda_{0}, \mu_{0}\right.$ as in Lemma 4.1), the functional

$$
J:\left[\lambda \frac{u_{*}}{4}, \lambda \frac{\underline{\sigma}}{c_{1}}\right] \times\left[\mu \frac{v_{*}}{4}, \mu \frac{\underline{b}}{c_{2}}\right] \rightarrow \mathbb{R}
$$


is continuously Fréchet differentiable. (Here the pair $\left(u_{*}, v_{*}\right)$ is again the unique positive solution of system (2.8).)

Proof. Let $\left(d_{1}, d_{2}\right)$ be an admissible control in

$$
\left[\lambda \frac{u_{*}}{4}, \lambda \frac{\underline{\sigma}}{c_{1}}\right] \times\left[\mu \frac{v_{*}}{4}, \mu \frac{\underline{b}}{c_{2}}\right]
$$

and $f, g \in L^{\infty}(\Omega)$. Proposition 3.3 can be applied. Consequently,

$$
\lim _{\beta \rightarrow 0}(1 / \beta)\left\{J\left(d_{1}+\beta f, d_{2}+\beta g\right)-J\left(d_{1}, d_{2}\right)\right\}=J^{\prime}\left(d_{1}, d_{2}\right)(f, g),
$$

where

$$
\begin{aligned}
J^{\prime}\left(d_{1}, d_{2}\right)(f, g) & =\int_{\Omega} \lambda \xi d_{1}+\mu \eta d_{2}+\lambda u f+\mu v g-2 d_{1} f-2 d_{2} g \\
& =\int_{\Omega}\left[\lambda u(1-r)-2 d_{1}\right] f+\left[\mu v(1-s)-2 d_{2}\right] g
\end{aligned}
$$

where $(r, s)$ is defined as in Theorem 3.5. The boundedness of terms in (4.1) gives us the continuity of linear operator $J^{\prime}\left(d_{1}, d_{2}\right)$. Now, take a sequence $\left(e_{1}^{n}, e_{1}^{n}\right)$ in

$$
\left[\lambda \frac{u_{*}}{4}, \lambda \frac{\underline{\sigma}}{c_{1}}\right] \times\left[\mu \frac{v_{*}}{4}, \mu \frac{\underline{b}}{c_{2}}\right]
$$

with $\left(e_{1}^{n}, e_{2}^{n}\right) \rightarrow\left(e_{1}, e_{2}\right)$. By similar arguments to those applied in Proposition 3.3, one obtains $\left(u^{n}, v^{n}\right) \rightarrow(u, v)$ and $\left(r^{n}, s^{n}\right) \rightarrow(r, s)$ in $H^{1}(\Omega) \cap L^{\infty}(\Omega)$. Therefore,

$$
J:\left[\lambda \frac{u_{*}}{4}, \lambda \frac{\underline{\sigma}}{c_{1}}\right] \times\left[\mu \frac{v_{*}}{4}, \mu \frac{\underline{b}}{c_{2}}\right] \rightarrow \mathbb{R}
$$

is continuously differentiable.

Remark 4.3. It is clear that, under the hypotheses of the previous proposition, functions

$$
u, v:\left[\lambda \frac{u_{*}}{4}, \lambda \frac{\underline{\sigma}}{c_{1}}\right] \times\left[\mu \frac{v_{*}}{4}, \mu \frac{\underline{b}}{c_{2}}\right] \rightarrow L^{\infty}(\Omega) \times L^{\infty}(\Omega)
$$

are Lipschitz continuous. This is a consequence of the Gateaux differentiability of $(u, v)$, the uniform estimates for $(\xi, \eta)$ with respect to $\left(d_{1}, d_{2}\right)$, and standard regularity arguments for elliptic equations. Let $L$ be a common Lipschitz constant for $u$ and $v$.

Lemma 4.4. Consider hypotheses (2.7), (3.7) and taking $0<\lambda<\lambda_{0}$, $0<\mu<\mu_{0}$ (the values $\lambda_{0}$ and $\mu_{0}$ determined in Lemma 4.1) and $\left(u_{*}, v_{*}\right)$ the positive solution of system (2.8). Then the functions

$$
r, s:\left[\lambda \frac{u_{*}}{4}, \lambda \frac{\underline{\sigma}}{c_{1}}\right] \times\left[\mu \frac{v_{*}}{4}, \mu \frac{\underline{b}}{c_{2}}\right] \rightarrow L^{\infty}(\Omega) \times L^{\infty}(\Omega),
$$

defined in Theorem 3.5, are locally Lipschitz continuous. 
Proof. Choose $k>0$ small enough for the following inequality to be verified

$$
\left(\bar{\sigma}-c_{1} u_{*}+\bar{b}-c_{2} v_{*}\right)^{2}<4\left(c_{1}\left(2 u_{*}+v_{*}\right)-k\right)\left(c_{2}\left(u_{*}+2 v_{*}\right)-k\right)
$$

Then system (3.9) may be rewritten as

$$
\begin{gathered}
(-\Delta+k) r+\left[d_{1}+c_{1}(2 u+v)-k\right] r-\frac{\mu}{\lambda}\left(b-c_{2} v\right) s=d_{1}, \quad \text { in } \Omega \\
(-\Delta+k) s+\left[d_{2}+c_{2}(u+2 v)-k\right] s-\frac{\lambda}{\mu}\left(\sigma-c_{1} u\right) r=d_{2}, \quad \text { in } \Omega \\
\frac{\partial r}{\partial \nu}=\frac{\partial s}{\partial \nu}=0, \quad \text { on } \partial \Omega
\end{gathered}
$$

or, equivalently,

$$
\left(\begin{array}{l}
r \\
s
\end{array}\right)=(-\Delta+k)^{-1}\left(\begin{array}{l}
{\left[-d_{1}-c_{1}(2 u+v)+k\right] r+\frac{\mu}{\lambda}\left(b-c_{2} v\right) s+d_{1}} \\
{\left[-d_{2}-c_{2}(u+2 v)+k\right] s+\frac{\lambda}{\mu}\left(\sigma-c_{1} u\right) r+d_{2}}
\end{array}\right)=F\left(r, s, d_{1}, d_{2}\right)
$$

The function $F$ is linear and continuous with respect to $r$ and $s$, so $F$ is continuously Frechet differentiable in $(r, s)$. Moreover, $F$ is Lipschitz continuous with respect to the other variables $\left(d_{1}, d_{2}\right)$. The implicit function theorem ensures that the map $\left(d_{1}, d_{2}\right) \mapsto$ $(r, s)$ is locally Lipschitz continuous.

Theorem 4.5. Assume hypotheses (2.7), (3.7) and $k_{1} \lambda<\mu<k_{2} \lambda$ for $k_{1}, k_{2}$ fixed positive constants (as in Lemma 4.1). Then there exist $\lambda_{1}, \mu_{1}>0$ such that if $0<\lambda<$ $\lambda_{1}, 0<\mu<\mu_{1}$, there exists a unique optimal control.

Proof. Define

$$
K=\left\{\left(d_{1}, d_{2}\right) \in\left[\lambda \frac{u_{*}}{4}, \lambda \frac{\underline{\sigma}}{c_{1}}\right] \times\left[\mu \frac{v_{*}}{4}, \mu \frac{\underline{b}}{c_{2}}\right]:\left(d_{1}, d_{2}\right) \text { is an optimal control }\right\},
$$

where $\left(u_{*}, v_{*}\right), \lambda$ and $\mu$ are taken as in Lemma 4.1. Let us begin by showing that $K \subset$ $L^{\infty}(\Omega) \times L^{\infty}(\Omega)$ is precompact. In fact, take $\left(d_{1}^{n}, d_{2}^{n}\right)$ a sequence of elements of $K$. From Theorem 3.5 and Lemma 4.1 we deduce that

$$
\begin{aligned}
& d_{1}^{n}=\frac{1}{2} \lambda u^{n}\left(1-r^{n}\right), \\
& d_{2}^{n}=\frac{1}{2} \mu v^{n}\left(1-s^{n}\right) .
\end{aligned}
$$

However, sequences $\left(u^{n}, v^{n}\right)$ and $\left(r^{n}, s^{n}\right)$ admit subsequences which converge in $C^{1, \alpha}(\bar{\Omega})$, $\alpha \in(0,1)$, in particular in $L^{\infty}(\Omega)$ (recall that both sequences are bounded in $W^{2, p}(\Omega)$, $p \in(1, \infty))$. Consider the compact set $\overline{c o}(K)$, the closed convex hull of $K$. The function $\overline{\mathrm{co}}(K) \rightarrow L^{\infty}(\Omega) \times L^{\infty}(\Omega),\left(d_{1}, d_{2}\right) \longmapsto(u(1-r), v(1-s))$ is Lipschitz continuous, $L$ again denoting its Lipschitz constant. Take $\lambda_{1}<\min \left\{\lambda_{0},(1 / L)\right\}, \mu_{1}<\min \left\{\mu_{0},(1 / L)\right\}$. In this situation we will prove that $J^{\prime}: \overline{c o}(K) \rightarrow\left(L^{\infty}(\Omega) \times L^{\infty}(\Omega)\right)^{*}$, defined as

$$
J^{\prime}\left(d_{1}, d_{2}\right)(f, g)=\int_{\Omega}\left[\lambda u(1-r)-2 d_{1}\right] f+\left[\mu v(1-s)-2 d_{2}\right] g, \quad \forall f, g \in L^{\infty}(\Omega),
$$


is strictly monotone, that is

$$
\left\langle J^{\prime}\left(d_{1}, d_{2}\right)-J^{\prime}\left(\bar{d}_{1}, \bar{d}_{2}\right),\left(d_{1}, d_{2}\right)-\left(\bar{d}_{1}, \bar{d}_{2}\right)\right\rangle<0, \quad \forall\left(d_{1}, d_{2}\right), \quad\left(\bar{d}_{1}, \bar{d}_{2}\right) \in \overline{\operatorname{co}}(K) .
$$

Consequently, $J$ is strictly concave on $\overline{\mathrm{co}}(K)$, and, therefore, $K$ reduces to a singleton. Let us see the latter. The choice of $\lambda$ and $\mu$ implies

$$
\begin{aligned}
(-2+\lambda L)\left|d_{1}-\bar{d}_{1}\right|^{2}+(-2+\mu L)\left|d_{2}-\bar{d}_{2}\right|^{2}+\left|d_{1}-\bar{d}_{1}\right|\left|d_{2}-\bar{d}_{2}\right| L(\lambda+\mu) \\
<-\left|d_{1}-\bar{d}_{1}\right|^{2}-\left|d_{2}-\bar{d}_{2}\right|^{2}+2\left|d_{1}-\bar{d}_{1}\right|\left|d_{2}-\bar{d}_{2}\right| \leqslant 0
\end{aligned}
$$

and it follows that

$$
\left\langle J^{\prime}\left(d_{1}, d_{2}\right)-J^{\prime}\left(\bar{d}_{1}, \bar{d}_{2}\right),\left(d_{1}, d_{2}\right)-\left(\bar{d}_{1}, \bar{d}_{2}\right)\right\rangle<0,
$$

for all $\left(d_{1}, d_{2}\right),\left(\bar{d}_{1}, \bar{d}_{2}\right) \in \overline{\mathrm{co}}(K)$. Obviously, the above inequality implies that there is only one point in $K$.

Example 4.6. Consider the problem of control where $\sigma \equiv b$ are constant functions, $\delta_{1}=\delta_{2}$, and $c_{1}=c_{2}$. In this case, if $\sigma>\delta$, Theorem 2.2 may be applied with $u_{*}=$ $(\sigma-\delta) / 2 c=v_{*}$ as lower solutions. If, in addition $2 \delta<\sigma$, condition (3.7) holds true, and, consequently, if $\lambda, \mu$ are small enough, the unique optimal control $\left(d_{1}, d_{2}\right) \in C_{\delta} \times C_{\delta}$ is described by Corollary 3.6 .

Acknowledgements. J.A.M. was supported in part by the Dirección General de Enseñanza Superior, Ministerio de Educación y Ciencia (Spain), under grant no. PB951190 and by EEC contract, Human Capital and Mobility programme ERBCHRXCT940494 .

\section{References}

1. H. AMANN, Fixed points equations and nonlinear eigenvalue problems in ordered Banach spaces, SIAM Rev. 18 (1976), 620-709.

2. A. Cañada, J. L. Gámez and J. A. Montero, Study of a nonlinear optimal control problem for diffusive nonlinear elliptic equations of logistic type, SIAM J. Control Optim. 36 (1998), 1171-1189.

3. F. H. Clarke, Optimization and nonsmooth analysis, Classics in Applied Mathematics, vol. 5 (SIAM, Philadelphia, 1990).

4. F. J. Correa AND M. A. Souto, On maximun principles for cooperative elliptic systems via fixed point index, Nonlinear Analysis 26 (1996), 997-1006.

5. I. Ekeland and R. Teman, Convex analysis and variational problems, Studies in Mathematics and its Applications, vol. 1 (North-Holland, Amsterdam, (1976).

6. J. L. GÁmez AND J. A. MONTERo, Uniqueness of the optimal control for a LotkaVolterra control problem with a large crowding effect, ESSAIM: Control, Optimisation and Calculus of Variations 2 (1997), 1-12 (http://www.emath.fr/cocv/).

7. D. GILBARG AND N. S. TRUDINGER, Elliptic partial differential equations of second order, 2nd edn (Springer, Berlin, 1983).

8. H. He, A. Leung and S. STOJAnovic, Periodic optimal control for competing parabolic Volterra-Lotka-type systems, J. Comput. Appl. Math. 52 (1994), 199-217. 
9. M. A. KRASNOSEl'SKII, Positive solutions of operator equations (Noordhoff, Groningen, 1964).

10. A. LEUNG, Optimal harvesting-coefficient control of steady-state prey-predator diffusive Volterra-Lotka systems, Appl. Math. Optim. 31 (1995), 219-241.

11. A. LeUng AND S. STOJANOvic, Optimal control for elliptic Volterra-Lotka type equations, J. Math. Analysis Appl. 173 (1993), 603-619.

12. M. RENARd AND R. C. Rogers, An introduction to partial differential equations, Texts in Applied Mathematics, vol. 13 (Springer, Berlin, 1993).

13. D. H. SATTINGER, Monotone methods in nonlinear elliptic and parabolic boundary value problems, Indiana Univ. Math. J. 21 (1972), 979-1000. 